\section{Prevalência de vacinação contra a influenza em idosos brasileiros com doenças crônicas}

\author{
Prevalence of influenza vaccination in elderly \\ Brazilian with chronic diseases
}

\section{Prevalencia de la vacunación contra la gripe en ancianos brasileños con enfermedades crónicas}

COMUNICAÇÃO BREVE

BRIEF COMMUNICATION
Aldiane Gomes de Macedo Bacurau 1

Priscila Maria Stolses Bergamo Francisco 1

doi: $10.1590 / 0102-311 \times 00230518$

\section{Resumo}

O objetivo deste estudo foi estimar a prevalência de vacinação contra a influenza em idosos brasileiros, segundo doenças crônicas específicas. Foram considerados os indivíduos com 60 anos ou mais $(n=23.815)$ participantes da Pesquisa Nacional de Saúde (PNS), realizada em 2013. Estimaram-se as prevalências de vacinação contra a influenza e os respectivos intervalos de 95\% de confiança (IC95\%). As associações foram verificadas pelo teste quiquadrado (Rao-Scott) considerando-se um nível de 5\% de significância. A prevalência de vacinação contra a influenza nos idosos foi de 73, 1\% (IC95\%: $72,0-74,1)$ e não houve diferença significativa nas prevalências entre os sexos $(p=0,237)$. Observaram-se diferenças estatisticamente significativas nas prevalências para os idosos que referiram hipertensão arterial $75 \%(p<0,001)$, diabetes mellitus 76,5\% ( $p=0,009)$, doenças do coração 79,2\% $(p<0,001) e$ doença no pulmão ou doença pulmonar obstrutiva crônica (DPOC) $87 \%$ $(p=0,001)$. Os resultados mostraram baixa prevalência de vacinação nos idosos com algumas condições crônicas específicas que possuem recomendação formal para receber a vacina, sugerindo a necessidade de que as campanhas de vacinação extrapolem o recorte etário (idosos) e sejam mais bem direcionadas para estes subgrupos específicos.

Vacinas Contra Influenza; Doença Crônica; Idoso; Inquéritos Epidemiológicos

\author{
Correspondência \\ A. G. M. Bacurau \\ Faculdade de Ciências Médicas, Universidade \\ Estadual de Campinas. \\ Rua Tessália Vieira de Camargo 126, sala 26, Campinas, SP \\ 13083-887, Brasil. \\ aldianemacedo@hotmail.com \\ 1 Faculdade de Ciências Médicas, Universidade Estadual de \\ Campinas, Campinas, Brasil.
}




\section{Introdução}

A influenza é uma doença respiratória aguda de distribuição global que constitui importante causa de morbimortalidade, especialmente nos indivíduos mais susceptíveis às complicações, como idosos e indivíduos com doenças crônicas 1,2,3,4,5,6. Durante as epidemias de influenza, a maioria dos casos graves, hospitalizações e óbitos são registrados nos grupos de risco, sobretudo, nos idosos 3,7.

A pneumonia (primária ou bacteriana secundária) é a complicação mais comum da doença, responsável pela maioria das hospitalizações e óbitos 1,2,3. No entanto, a influenza pode ocasionar o agravamento de doenças crônicas cardiovasculares, pulmonares, diabetes, desencadear crises de asma, infarto agudo do miocárdio (IAM), acidente vascular cerebral (AVC), miocardite, pericardite, insuficiência renal, falência de múltiplos órgãos, entre outras exacerbações de doenças e condições clínicas 1,2,4,8,9.

Além da recomendação da vacina pela Organização Mundial da Saúde (OMS), como estratégia de saúde pública para prevenir contra a influenza e suas complicações 3, outros órgãos também a indicam para idosos e indivíduos com doenças crônicas 5,10. Nos mais longevos, a vacina pode ser menos eficaz na prevenção da doença, mas reduz os quadros graves e óbitos 3. Estudos mostram a diminuição das complicações, internações hospitalares e mortes relacionadas à influenza, além de gastos com hospitalizações 9,10,11,12,13,14,15,16.

No Brasil, o Ministério da Saúde disponibiliza a vacina para os idosos, indivíduos com doença pulmonar crônica (doença pulmonar obstrutiva crônica - DPOC, asma), doenças cardíacas, diabetes, insuficiência renal, AVC, pessoas com imunossupressão por medicamentos ou doenças (HIV/aids, câncer) e outros grupos de risco ${ }^{4}$. Mesmo diante da recomendação e disponibilização da vacina para esses grupos, dados sobre a prevalência de vacinação na população brasileira com doenças crônicas são escassos. O objetivo deste estudo foi estimar as prevalências de vacinação contra a influenza em idosos brasileiros, segundo doenças crônicas específicas.

\section{Métodos}

Estudo realizado com dados de domínio público de idosos ( $\geq 60$ anos) que participaram da Pesquisa Nacional de Saúde (PNS, $\mathrm{n}=23.815$ ), inquérito nacional de base domiciliar, realizado no Brasil entre 2013 (Instituto Brasileiro de Geografia e Estatística. Pesquisa Nacional de Saúde 2013. https://ww2. ibge.gov.br/home/estatistica/populacao/pns/2013/default.shtm, acessado em 14/Mar/2018).

A população de estudo da PNS 2013 foi pessoas com idade $\geq 18$ anos, residentes em domicílios particulares permanentes, localizados em área urbana ou rural brasileira. A amostra representativa da população foi obtida por amostragem conglomerada em três estágios, com estratificação das unidades primárias. As unidades primárias de amostragem constituíram-se de setores censitários ou conjunto de setores, os domicílios (selecionados por amostra aleatória simples) formaram as unidades de segundo estágio e os moradores ( $\geq 18$ anos), as unidades de terceiro estágio. Em cada domicílio, foi selecionado aleatoriamente um morador para responder ao questionário individual. Mais detalhes sobre o método da PNS estão disponíveis em publicações prévias 17,18.

As variáveis utilizadas foram obtidas nos módulos sobre características gerais e de educação dos moradores e doenças crônicas. No módulo sobre a saúde dos idosos, obtiveram-se informações sobre a vacinação contra a influenza nos 12 meses anteriores à pesquisa.

Estimaram-se as prevalências de vacinação contra a influenza e os respectivos intervalos de 95\% de confiança (IC95\%), considerando-se as doenças crônicas específicas (única doença). As associações foram verificadas pelo teste qui-quadrado (Rao-Scott) com nível de 5\% de significância. As análises foram realizadas no Stata 14.0 (https://www.stata.com), considerando-se as ponderações do desenho amostral.

A PNS foi aprovada pela Comissão Nacional de Ética em Pesquisa (processo no 328.159, de 26 de junho de 2013) e todos os participantes assinaram Termo de Consentimento Livre e Esclarecido. Os microdados da pesquisa estão disponíveis na página eletrônica do Instituto Brasileiro de Geografia e Estatística (IBGE. Pesquisa Nacional de Saúde 2013. https://ww2.ibge.gov.br/home/estatistica/ populacao/pns/2013/default.shtm, acessado em 14/Mar/2018). 


\section{Resultados}

A média de idade da população idosa foi de 69,9 anos (IC95\%: 69,7-70,1) e a maioria era mulheres (56,4\%; IC95\%: 55,6-57,2). A prevalência de vacinação contra a influenza nos idosos foi de $73,1 \%$ (IC95\%: 72,0-74,1), sem diferença entre os sexos ( $\mathrm{p}=0,237)$.

Para o total dos idosos, encontraram-se diferenças significativas nas prevalências de vacinação para aqueles com hipertensão arterial 75\% ( $\mathrm{p}<0,001)$, diabetes $76,5 \%(\mathrm{p}=0,009)$, doenças do coração $79,2 \%(\mathrm{p}<0,001)$ e doença no pulmão ou DPOC $87 \%(\mathrm{p}=0,001)$. Para AVC (59,7\%), asma (66\%), artrite ou reumatismo (71\%), insuficiência renal (64,8\%) e câncer ( $73,5 \%)$ não houve diferença estatisticamente significativa nas prevalências de vacinação $(\mathrm{p}>0,05)$ (Tabela 1$)$.

Para o subgrupo das doenças do coração, a prevalência de vacinação nos idosos com IAM foi de $83,4 \%(\mathrm{n}=40)$ e insuficiência cardíaca $92,9 \%(\mathrm{n}=36)$.

$\mathrm{Na}$ análise das doenças específicas segundo o sexo, constataram-se diferenças significativas $(\mathrm{p}<$ $0,05)$ nas prevalências de vacinação para hipertensão arterial, asma, artrite ou reumatismo, doenças no pulmão ou DPOC e insuficiência renal (maiores no sexo feminino) e AVC (maior no masculino) (Figura 1).

\section{Discussão}

Os resultados apontaram prevalências de vacinação abaixo da meta estabelecida pelo Ministério da Saúde em 2013 (80\%) 4, exceto para doença no pulmão ou DPOC. No Brasil, existem poucos estudos sobre a vacinação contra a influenza em pessoas com condições clínicas específicas. Estudo identificou prevalência de vacinação de 56,7\% 19 em idosos com doença respiratória pulmonar crônica, e, no Município de São Paulo, em 2015, a prevalência foi de 59,2\% naqueles com diabetes 20. Para tais condições específicas, as prevalências encontradas neste estudo foram maiores.

Estudos internacionais, que incluíram idosos (idade $\geq 65$ anos), encontraram baixas prevalências 21,22 e coberturas 23 de vacinação contra a influenza em indivíduos com doenças crônicas. $\mathrm{Na}$ Polônia, em pacientes com câncer, a cobertura foi de $9 \%$, doenças cardiovasculares $32 \%$, insuficiência renal 34\% e doença pulmonar 58\% 23. Em pacientes com DPOC, na Espanha, a prevalência foi de $62,7 \%$ e diminuiu com o aumento da idade e gravidade da doença 24, já nos Estados Unidos foi de $53 \%$, a idade avançada foi fator predisponente à vacinação ( $66,7 \%$ naqueles com idade $\geq 65$ anos) 25 . Ressalta-se que esses estudos consideraram diferentes recortes etários, inviabilizando comparações diretas quanto às prevalências verificadas. Vários fatores podem influenciar a adesão à vacinação: não

\section{Tabela 1}

Prevalências de doenças crônicas e de vacinação contra a influenza nos idosos, segundo as doenças específicas. Pesquisa Nacional de Saúde, Brasil, 2013.

\begin{tabular}{lccccc}
\hline Variáveis & \multicolumn{2}{c}{ Prevalência da doença } & \multicolumn{2}{c}{ Prevalência de vacinação contra influenza } \\
& $\%$ & IC95\% & $\%$ & IC95\% & Valor de p \\
\hline Hipertensão arterial & 51,4 & $49,9-53,0$ & 75,0 & $72,4-77,4$ & $<0,001$ \\
Diabetes mellitus & 18,2 & $17,1-19,4$ & 76,5 & $69,4-82,4$ & 0,009 \\
Doenças do coração & 11,8 & $10,7-13,0$ & 79,2 & $72,3-84,7$ & $<0,001$ \\
AVC & 4,7 & $4,2-5,4$ & 59,7 & $42,1-75,1$ & 0,419 \\
Asma & 5,0 & $4,4-5,7$ & 66,0 & $53,2-76,8$ & 0,937 \\
Artrite ou reumatismo & 17,3 & $16,2-18,4$ & 71,0 & $65,0-76,3$ & 0,152 \\
Doenças no pulmão ou DPOC & 3,8 & $3,2-4,5$ & 87,0 & $75,7-93,5$ & 0,001 \\
Insuficiência renal & 2,7 & $2,3-3,2$ & 64,8 & $41,4-82,8$ & 0,881 \\
Câncer & 5,6 & $5,0-6,4$ & 73,5 & $61,5-82,9$ & 0,243 \\
\hline
\end{tabular}

AVC: acidente vascular cerebral; DPOC: doença pulmonar obstrutiva crônica; IC95\%: intervalos de 95\% de confiança. 


\section{Figura 1}

Prevalência de vacinação contra influenza em idosos com doenças crônicas, segundo o sexo. Pesquisa Nacional de Saúde, Brasil, 2013.

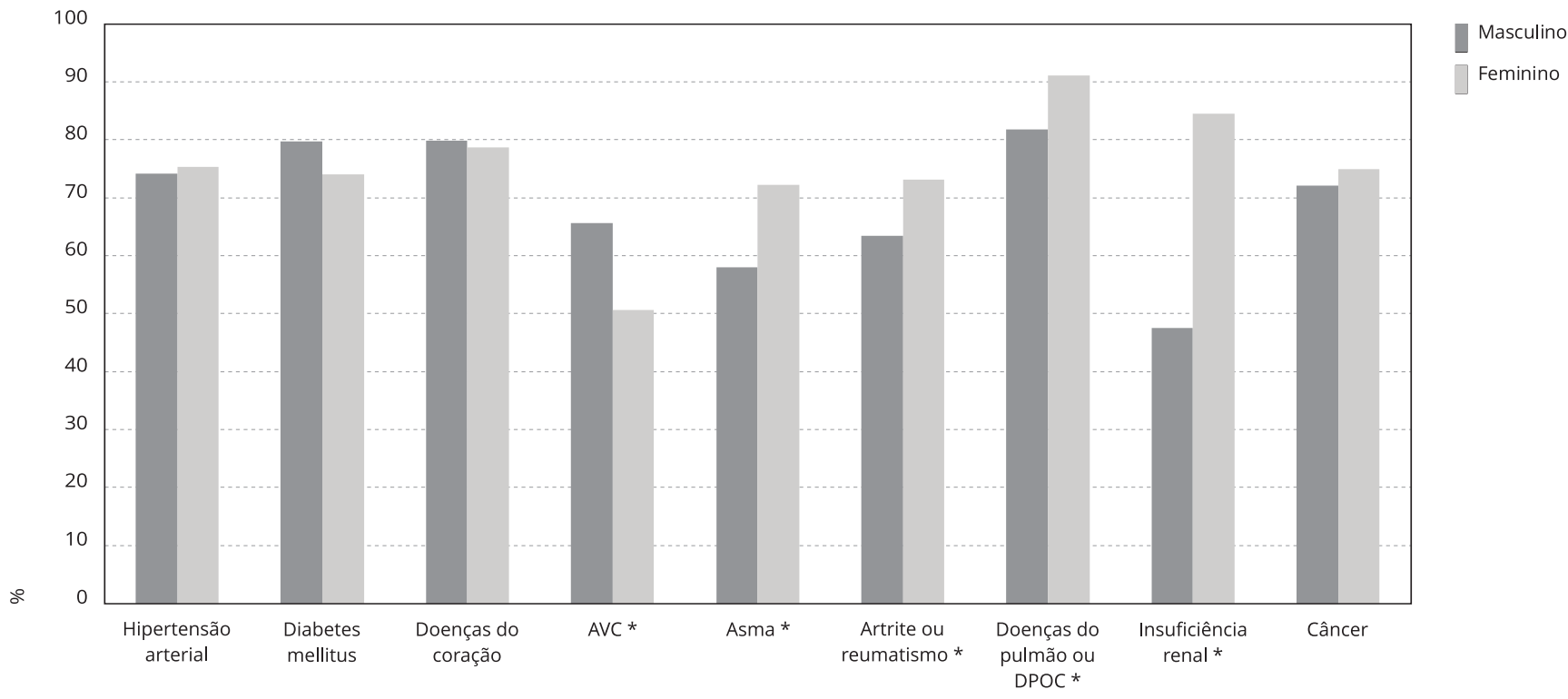

AVC: acidente vascular cerebral; DPOC: doença pulmonar obstrutiva crônica.

* Apresentou diferença estatisticamente significativa na prevalência de vacinação entre os sexos $(p<0,05)$

achar necessário 22 , falta de recomendação dos profissionais de saúde 23 e pouco conhecimento sobre a gravidade da influenza 23 têm sido reportados como motivos para a não vacinação de pessoas com doenças crônicas.

Deve se considerar as limitações inerentes aos estudos transversais, especialmente quanto à informação sobre doença referida (que depende do acesso aos serviços de saúde e diagnóstico prévio) Tendo em vista que a prevalência de doenças crônicas aumenta com a idade, a política de vacinação nesses grupos de risco deve ser incentivada, pois a vacina previne os casos graves da doença e óbitos em idosos e indivíduos com doenças crônicas 4,11,12,13,14,15,16,24.

\section{Conclusão}

Os resultados mostraram baixa prevalência de vacinação em idosos para algumas doenças crônicas específicas, sugerindo que as campanhas devem extrapolar o recorte etário e intensificar a abordagem da recomendação para tais subgrupos. 


\section{Colaboradores}

A. G. M. Bacurau participou na realização da análise dos dados, interpretação dos resultados e redação do manuscrito. P. M. S. B. Francisco colaborou com a análise dos dados, interpretação dos resultados, redação e revisão crítica do manuscrito.

\section{Informações adicionais}

ORCID: Aldiane Gomes de Macedo Bacurau (00000002-6671-2284); Priscila Maria Stolses Bergamo Francisco (0000-0001-7361-9961).

\section{Agradecimentos}

Agradecemos ao Ministério da Saúde (MS) e ao Instituto Brasileiro de Geografia e Estatística (IBGE) por disponibilizarem os dados dos participantes da Pesquisa Nacional de Saúde 2013 (PNS 2013). À Coordenação de Aperfeiçoamento de Pessoal de Nível Superior (Capes) pela concessão da bolsa de doutorado a A. G. M. Bacurau.

\section{Referências}

1. Paules C, Subbarao K. Influenza. Lancet 2017; 390:697-708.

2. Centers for Disease Control and Prevention. Flu symptoms \& complications. http://www. cdc.gov/flu/about/disease/complications.htm (acessado em 25/Jul/2018).

3. World Health Organization. Influenza (seasonal). http://www.who.int/mediacentre/fact sheets/fs211/en/ (acessado em 25/Out/18).

4. Coordenação Geral do Programa Nacional de Imunizações, Departamento de Vigilância Epidemiológica, Secretaria de Vigilância em Saúde, Ministério da Saúde. Campanha Nacional de Vacinação Contra a Influenza: informe técnico. 20a Ed. Brasília: Ministério da Saúde; 2018.

5. Centers for Disease Control and Prevention. Adults with chronic conditions: get vaccinated. https://www.cdc.gov/features/vaccin eschronicconditions/index.html (acessado em 05/Nov/2018).

6. Mertz D, Kim TH, Johnstone J, Lam PP, Science M, Kuster SP, et al. Populations at risk for severe or complicated influenza illness: systematic review and meta-analysis. BMJ 2013; 347:f5061.

7. Reed C, Chaves SS, Daily Kirley P, Emerson R, Aragon D, Hancock EB, et al. Estimating Influenza disease burden from population-based surveillance data in the United States. PLoS One 2015; 10:e0118369.

8. Estabragh ZR, Mamas MA. The cardiovascular manifestations of influenza: a systematic review. Int J Cardiol 2013; 167:2397-403.

9. Bricks LF, Carvalhanas TRMP, Domingues CMAS, Pereira SF, Bellei NCJ. Influenza em pacientes com doenças cardíacas crônicas: o que há de novo? J Health Biol Sci 2015; 3:16571.

10. European Centre for Disease Prevention and Control. Risk groups for severe influenza. https://ecdc.europa.eu/en/seasonal-influenza/ prevention-and-control/vaccines/risk-groups (acessado em 11/Nov/2018).

11. Wang CS, Wang ST, Lai CT, Lin LJ, Chou P. Impact of influenza vaccination on major cause-specific mortality. Vaccine 2007; 25:1196-203.

12. Nichol KL, Baken L, Nelson A. Relation between influenza vaccination and outpatient visits, hospitalization, and mortality in elderly persons with chronic lung disease. Ann Intern Med 1999; 130:397-403.

13. Wang IK, Lin CL, Chang YC, Lin PC, Liang CC, Liu YL, et al. Effectiveness of influenza vaccination in elderly diabetic patients: a retrospective cohort study. Vaccine 2013; 31:71824.

14. Clar C, Oseni Z, Flowers N, Keshtkar-Jahromi $\mathrm{M}$, Rees K. Influenza vaccines for preventing cardiovascular disease. Cochrane Database Syst Rev 2015; (5):CD005050. 
15. Casado I, Domínguez A, Toledo D, Chamorro J, Astray J, Egurrola M, et al. Repeated influenza vaccination for preventing severe and fatal influenza infection in older adults: a multicentre case-control study. CMAJ 2018; 190:E3-12.

16. Arriola C, Garg S, Anderson EJ, Ryan PA, George A, Zansky SM, et al. Influenza vaccination modifies disease severity among community-dwelling adults hospitalized with influenza. Clin Infect Dis 2017; 65:1289-97.

17. Szwarcwald CL, Malta DC, Pereira CA, Vieira MLFP, Conde WL, Souza Júnior PRB, et al. Pesquisa Nacional de Saúde no Brasil: concepção e metodologia de aplicação. Ciênc Saúde Colet 2014; 19:333-42.

18. Souza-Júnior PRB, Freitas MPS, Antonaci GA, Szwarcwald CL. Desenho da amostra da Pesquisa Nacional de Saúde 2013. Epidemiol Serv Saúde 2015; 24:207-16.

19. Bacurau AGM, Francisco PMSB. Prevalence of influenza vaccination in adults and elderly with chronic respiratory diseases. Cad Saúde Pública 2018; 34:e00194717.

20. Monteiro CN, Gianini RJ, Stopa SR, Segri NJ, Barros MBA, Cesar CLG, et al. Cobertura vacinal e utilização do SUS para vacinação contra gripe e pneumonia em adultos e idosos com diabetes autorreferida, no município de São Paulo, 2003, 2008 e 2015. Epidemiol Serv Saúde 2018; 27:e2017272.
21. Santaularia J, Hou W, Perveen G, Welsh E, Faseru B. Prevalence of influenza vaccination and its association with health conditions and risk factors among Kansas adults in 2013: a crosssectional study. BMC Public Health 2016; 16:185.

22. Vozoris NT, Lougheed MD. Influenza vaccination among Canadians with chronic respiratory disease. Respir Med 2009; 103:50-8.

23. Nitsch-Osuch A, Gołębiak I, Wyszkowska D, Rosińska R, Kargul L, Szuba B, et al. Influenza vaccination coverage among Polish patients with chronic diseases. Adv Exp Med Biol 2017; 968:19-34

24. Garrastazu R, Garcia-Rivero JL, Ruiz M, Helguera JM, Arenal S, Bonnardeux C, et al. Prevalence of influenza vaccination in chronic obstructive pulmonary disease patients and impact on the risk of severe exacerbations. Arch Bronconeumol 2016; 52:88-95.

25. Arabyat RM, Raisch DW, Bakhireva L. Influenza vaccination for patients with chronic obstructive pulmonary disease: implications for pharmacists. Res Social Adm Pharm 2018; 14:162-9. 


\section{Abstract}

This study aimed to estimate the prevalence of influenza vaccination in elderly Brazilians with specific chronic diseases. The sample included individuals 60 years or older $(n=23,815)$ participating in the National Health Survey (PNS) in 2013. The study estimated the prevalence rates for influenza vaccination and the respective $95 \%$ confidence intervals $(95 \% \mathrm{CI})$. The associations were verified with the chi-square test (Rao-Scott) with 5\% significance. Prevalence of influenza vaccination in the elderly was $73.1 \%$ (95\%CI: 72.0-74.1), and there was no significant difference in prevalence rates between men and women $(p=0.237)$. Statistically significant differences were observed in prevalence rates for elderly that reported arterial hypertension, $75 \%(p<0.001)$, diabetes mellitus, 76.5\% ( $p=0.009)$, cardiac disease, 79.2\% ( $p$ $<0.001$ ), and lung disease or chronic obstructive pulmonary disease $(C O P D), 87 \%(p=0.001)$. The results showed low prevalence of vaccination in elderly with some specific chronic diseases for whom there is formal recommendation to receive the vaccine, suggesting the need for a vaccination campaign to extrapolate the age cutoff (elderly) and better targeting to these specific subgroups.

Influenza Vaccines; Chronic Disease; Aged; Health Surveys

\section{Resumen}

El objetivo de este estudio fue estimar la prevalencia de vacunación contra la gripe en ancianos brasileños, según enfermedades crónicas especificas. Se consideraron individuos de 60 años o más $(n=23.815)$, participantes en la Encuesta $\mathrm{Na}$ cional de Salud (PNS, por sus siglas en portugués), realizada en 2013. Se estimaron las prevalencias de vacunación contra la gripe y sus respectivos intervalos de 95\% de confianza (IC95\%). Las asociaciones se verificaron mediante el test chicuadrado (Rao-Scott), considerándose un nivel del 5\% de significancia. La prevalencia de vacunación contra la gripe en los ancianos fue de un 73,1\% (IC95\%: 72,0-74,1) y no hubo una diferencia significativa en las prevalencias entre sexos $(p=$ $0,237)$. Se observaron diferencias estadísticamente significativas en las prevalencias con los ancianos que informaron de hipertensión arterial $75 \%(p<$ $0,001)$, diabetes mellitus $76,5 \%(p=0,009)$, enfermedades del corazón $79,2 \%(p<0,001)$ y enfermedad del pulmón o enfermedad pulmonar obstructiva crónica (EPOC) $87 \%(p=0,001)$. Los resultados mostraron una baja prevalencia de vacunación en los ancianos con algunas condiciones crónicas de salud específicas, que cuentan con una recomendación formal para recibir la vacuna, sugiriendo la necesidad de que las campañas de vacunación extrapolen la acotación por franja de edad (ancianos) y sean dirigidas especificamente mejor hacia estos subgrupos.

Vacunas Contra la Influenza; Enfermedad Crónica; Anciano; Encuestas Epidemiológicas
Recebido em 30/Nov/2018

Versão final reapresentada em 12/Fev/2019

Aprovado em 28/Fev/2019 